\title{
Editorial
}

\section{Pediatric Hemato-Oncology}

\author{
Pascal Chastagner \\ Guest Editor
}

Professor, MD, PhD, Head of Department of Pediatric Hemato-Oncology of CHRU Nancy, Children's Hospital, Vandoeuvre, France

Pediatric cancers, like all the diseases arising in children, bring together numerous very rare diseases which make each of them "orphan diseases". However, cancer remains the first disease-related cause of death in most of the middle-high income countries with an incidence of approximately 1 in 7000 children younger than 19 years of age.

Childhood cancer survival outcomes have greatly benefited from extensive implementation of national, and more and more often, international clinical trials. However, about $20 \%$ of children still die from their malignancies and at least $50 \%$ of them suffer from long-term sequelae in adulthood. Consequently, pediatric oncologists have the double challenge of curing the disease and preventing as many sequelae as possible, which represent a major problem when treating a child in full growth by chemo- and above all radiotherapy.

Nowadays, the role of molecular biology and genetics has transformed the way of diagnosis, treatment and prevention of cancer, particularly by allowing the selection of "good prognosis" patients for whom the intensity of treatment could be decreased and, on the contrary, "high risk" patients for whom the treatments are intensified. Moreover, in order to increase cure rate and decrease the frequency and severity of sequelae, novel drugs targeting oncogenic drivers are currently investigated in pediatric oncology, similarly to the adult cancer treatment approach. This turning point in our research fields and practice has profoundly changed the place of clinical observations in publications. Indeed, it has become very difficult, if not impossible, to publish clinical case reports which are nevertheless useful and needed in countless difficult situations that clinicians may have never encountered in their practice. Still two decades ago, a large place was proposed for case reports or small sample studies in cancer journals, particularly in pediatric cancer journals, which contributed in part to the great improvement of pediatric oncologists' knowledge and practice, as well as treatment quality.

Furthermore, while progress has been made in the treatment of most childhood cancers, some malignancies, too rare to be included in clinical trials, have remained very difficult to treat and case reports provide an excellent way of sharing our experience.

I would like to express here my deep appreciation to Prof. M. Danciu and the "Archive of Clinical Cases" for dedicating a special issue on pediatric Hemato-oncology and giving the opportunity to pediatric oncologists to benefit from shared expertise.

The selected case reports offer a great panel of diagnosis and/or treatment challenges, which, I hope, could help clinicians in their daily practice and give the motivation to younger practitioners to continue and develop clinical research. 